\title{
Commentary: We can prevent postesophagectomy atrial fibrillation, but should we?
}

\author{
Omar F. Hassan, MD, and Benny Weksler, MBA, MD
}

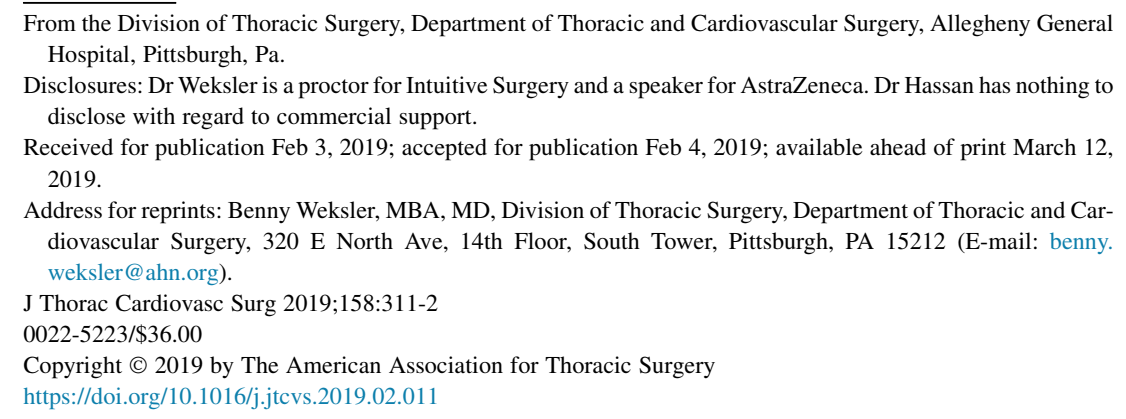

The postoperative care of patients who have undergone esophagectomy is complex and offers many confounding challenges for the thoracic surgeon. Atrial fibrillation is a common complication that frequently requires intervention $^{1}$ and is associated with increased anastomotic leaks, hospital length of stay, and mortality. ${ }^{2}$ Amiodarone is commonly used for the treatment of postoperative atrial fibrillation and has been shown to reduce the incidence of atrial fibrillation in thoracic surgical patients, ${ }^{3,4}$ including in patients undergoing esophagectomy. ${ }^{5}$ Despite preventing atrial fibrillation, amiodarone has not been associated with decreased in length of stay., ${ }^{3,5-7}$

In this issue of the Journal, Tisdale and colleagues ${ }^{8}$ expand on their previous work looking at prophylactic amiodarone's effect on postoperative atrial fibrillation after esophagectomy. In this retrospective review, they propensity matched 220 pairs out of a total 309 patients who underwent esophagectomy, 110 of whom received prophylactic intravenous amiodarone after esophagectomy and 110 of whom did not. The study presents us with several important findings. In concordance with their previous work, Tisdale and colleagues ${ }^{8}$ found prophylactic amiodarone to decrease the incidence of postoperative atrial fibrillation. The amiodarone group, however, had higher incidences of hypotension requiring treatment, bradycardia, and prolonged corrected QT interval. There were no differences in pulmonary complications, hospital length of stay, or mortality. The number of patients needed to treat to prevent a single episode of atrial fibrillation was 6.9, but the number of patients treated needed to see harm was only 4.8. In patients who had atrial fibrillation develop, the rate of conversion to normal sinus was the same in both groups, and there was no difference in the conversion rate according to whether patients received amiodarone or other alternative therapies.

Tisdale and colleagues ${ }^{8}$ illuminate some of the risks associated with the administration of prophylactic amiodarone

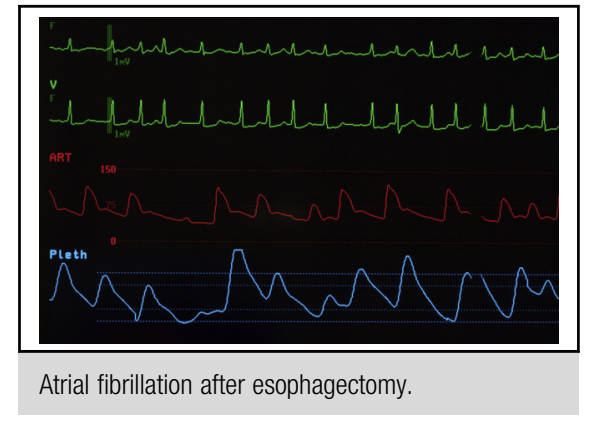

Central Message

Prophylactic amiodarone after esophagectomy prevents atrial fibrillation, but it is associated with higher incidences of hypotension, bradycardia, and prolonged QT interval.

See Article page 301. in patients undergoing esophagectomy. With careful propensity matching, Tisdale and colleagues ${ }^{8}$ did not find an association between amiodarone prophylaxis and hospital length of stay or mortality, but amiodarone increased complications. For most of the study period, the clinical pathway of Tisdale and colleagues ${ }^{8}$ was quite conservative, with a discharge target at postoperative day 10 , whereas the incidence of atrial fibrillation in the patients not receiving prophylaxis plateaued at postoperative day 6 . It is plausible that a more aggressive pathway with a discharge day target at or sooner than 7 days amiodarone could affect hospital length of stay.

The current work of Tisdale and colleagues ${ }^{8}$ is important and demonstrates that although prophylactic amiodarone decreases postoperative atrial fibrillation, it increases the risk of hypotension needing treatment, bradycardia, and a prolonged corrected QT. Amiodarone may have harmed more patients than it helped, as illustrated by the number of patients needed to treat to see benefit relative to the number of patients needed to treat to see harm. These findings make a compelling argument that prophylactic amiodarone should be administered rarely and only after significant assessment of individual patient risk. There is no question that atrial fibrillation can be prevented. A better question is whether we should prevent it with the agents currently available. Larger prospective, randomized trials are needed 
to settle the role of prophylactic amiodarone for patients undergoing esophagectomy.

\section{References}

1. Mc Cormack O, Zaborowski A, King S, Healy L, Daly C, O'Farrell N, et al. Newonset atrial fibrillation post-surgery for esophageal and junctional cancer: incidence, management, and impact on short- and long-term outcomes. Ann Surg. 2014;260:772-8; discussion 778 .

2. Chen LT, Jiang CY. Impact of atrial arrhythmias after esophagectomy on recovery: a meta-analysis. Medicine (Baltimore). 2018;97:e10948.

3. Tisdale JE, Wroblewski HA, Wall DS, Rieger KM, Hammoud ZT, Young JV, et al. A randomized trial evaluating amiodarone for prevention of atrial fibrillation after pulmonary resection. Ann Thorac Surg. 2009;88:886-93; discussion 894-5.
4. Crystal E, Connolly SJ, Sleik K, Ginger TJ, Yusuf S. Interventions on prevention of postoperative atrial fibrillation in patients undergoing heart surgery: a metaanalysis. Circulation. 2002;106:75-80.

5. Tisdale JE, Wroblewski HA, Wall DS, Rieger KM, Hammoud ZT, Young JV, et al. A randomized, controlled study of amiodarone for prevention of atrial fibrillation after transthoracic esophagectomy. J Thorac Cardiovasc Surg. 2010;140:45-51.

6. Guarnieri T, Nolan S, Gottlieb SO, Dudek A, Lowry DR. Intravenous amiodarone for the prevention of atrial fibrillation after open heart surgery: the Amiodarone Reduction in Coronary Heart (ARCH) trial. J Am Coll Cardiol. 1999;34:343-7.

7. Mitchell LB, Exner DV, Wyse DG, Connolly CJ, Prystai GD, Bayes AJ, et al. Prophylactic Oral Amiodarone for the Prevention of Arrhythmias that Begin Early After Revascularization, Valve Replacement, or Repair: PAPABEAR: a randomized controlled trial. JAMA. 2005;294:3093-100.

8. Tisdale JE, Jaynes HA, Watson MR, Corya AL, Shen C, Kesler KA. Amiodarone for prevention of atrial fibrillation following esophagectomy. J Thorac Cardiovasc Surg. 2019;158:301-10.e1. 